\title{
O uso da eletroconvulsoterapia no Instituto de Psiquiatria da Universidade Federal do Rio de Janeiro no período de 2005 a 2007
}

\author{
Use of electroconvulsive therapy at Instituto de Psiquiatria, Universidade Federal \\ do Rio de Janeiro, from 2005 to 2007
}

\section{Daniele Lauriano Pastore ${ }^{1}$, Leandro Marchetti Bruno ${ }^{1}$, Antonio Egidio Nardi², Allan Gonçalves Dias $^{3}$}

${ }^{1}$ Acadêmico(a) de Medicina, Universidade Federal do Rio de Janeiro (UFRJ), Rio de Janeiro, RJ. ${ }^{2}$ Livre-docente. Professor associado, Instituto de Psiquiatria, Faculdade de Medicina, UFRJ. ${ }^{3}$ Psiquiatra, Instituto de Psiquiatria, IPUB-UFRJ.

Este estudo foi realizado no Laboratório de Pânico e Respiração, Instituto de Psiquiatria, Universidade Federal do Rio de Janeiro (IPUB-UFRJ), Rio de Janeiro, RJ.

\section{Resumo}

Introdução: Este estudo teve como objetivo comparar os dados atuais sobre a eletroconvulsoterapia (ECT) no Instituto de Psiquiatria da Universidade Federal do Rio de Janeiro a dados de estudos anteriores e a pesquisas relacionadas ao tema em outros países.

Método: Foram analisados prontuários de junho de 2005 a junho de 2007, reunindo um total de 69 pacientes que realizaram ECT no período e avaliadas as seguintes categorias: sexo, idade, diagnóstico, indicação e número de aplicações de ECT realizados por paciente. Além disso, foram coletadas informações subjetivas sobre a remissão dos sintomas na evolução desses pacientes.

Resultados: Observou-se um predomínio do sexo feminino (71\%). A média de idade dos pacientes foi de 41,3 anos $(p=13,7)$. Os principais diagnósticos encontrados foram esquizofrenia $(49,3 \%)$ e transtorno afetivo bipolar $(27,5 \%)$. A indicação mais comum foi a heteroagressividade, seguida da tentativa de suicídio e da auto-agressividade. A média de número de ECT realizados por paciente foi de 8,2. De um modo geral, houve remissão dos sintomas no curto prazo após a realização desse método de tratamento.

Conclusão: As aplicações da ECT no Instituto de Psiquiatria da Universidade Federal do Rio de Janeiro estão de acordo com os critérios e recomendações da Associação Mundial de Psiquiatria e com o modelo utilizado nos países comparados. Houve uma manutenção geral dos padrões utilizados nessa terapêutica no instituto. Além disso, a ECT se mostrou um bom método para remissão de sintomas graves no curto prazo.

Descritores: Eletroconvulsoterapia, psiquiatria, eficácia, epidemiologia, tratamento.

\begin{abstract}
Introduction: The objective of this study was to compare current data on electroconvulsive therapy (ECT) at Instituto de Psiquiatria, Universidade Federal do Rio de Janeiro to previous studies and related research in other countries.

Method: Medical records of 69 patients who were submitted to ECT were analyzed from June 2005 to June 2007, evaluating the following categories: sex, age, diagnostic, indication and number of ECT sessions each patient received. In addition, subjective information about remission of symptoms was collected in the evolution of these patients.

Results: There was a preponderance of females $(71 \%)$. Mean age of patients was 41.3 years $(\mathrm{p}=13.7)$. The most frequent diagnoses were schizophrenia (49.3\%) and bipolar disorder (27.5\%). The most common indication was interpersonal violence, followed by suicide attempt and self-injury. Mean number of ECT sessions received by each patient was 8.2. In general there was remission of symptoms in the short term after using this method.
\end{abstract}

\section{Correspondência:}

Daniele Lauriano Pastore, Rua Visconde de Pirajá, 407/702, Ipanema, CEP 22410-003, Rio de Janeiro, RJ. E-mail: danielepastore@ufrj.br Não há conflitos de interesse associados à publicação deste artigo. 
Conclusion: Use of ECT at Instituto de Psiquiatria, Universidade Federal do Rio de Janeiro is in agreement with the criteria and recommendations of the World Psychiatry Association and with the model used in other countries. There was general maintenance of the standards used for this treatment at Instituto de Psiquiatria. Furthermore, ECT proved to be a good method for remission of severe symptoms in the short term.

Keywords: Electroconvulsive therapy, psychiatry, efficacy, epidemiology, treatment.

\section{Introdução}

A carência de conhecimento físiopatológico e de tratamentos eficazes na psiquiatria do início do século $\mathrm{XX}$ era tal, que muitos cientistas passaram a criar métodos dos mais variados, baseados puramente na observação de poucos casos e sem grupo controle. Desse modo, a malarioterapia iniciada por Wagner-Jauregg em $1927^{1}$, a insulinoterapia desenvolvida por Meduna em $1933^{1}$ e a lobotomia realizada por Egas Moniz em $1935^{1}$ foram formuladas como tentativas de alívio dos sintomas psicóticos, mas certamente induziam ao paciente um sofrimento maior do que sua própria doença. Ainda assim, o Nobel da Medicina foi atribuído a WagnerJauregg e a Egas Moniz, as únicas representações da psiquiatria nessa premiação.

A eletroconvulsoterapia (ECT) iniciada por Cerletti $\&$ Bini $^{1}$ em 1938 veio como uma alternativa de tratamento aos pacientes psicóticos. Mas foi, desde o início, cercada de críticas e recusas ao seu uso. Há, até hoje, a comparação, sem nenhum embasamento científico, da indução de convulsões nos pacientes à punição por métodos de tortura, à geração de dor, a fortes efeitos colaterais e a seu uso compulsório, sem consentimento. No entanto, a eficácia desse tratamento ${ }^{2}$ faz com que ele venha sobrevivendo a toda a polêmica a seu respeito, ainda que os psicofármacos, desde a década de 50 , tenham trazido uma redução progressiva de seu uso.

A Associação Psiquiátrica Americana (American Psychiatric Association, APA) ${ }^{3}$ e a Associação Brasileira de Psiquiatria ${ }^{4}$ estão envolvidas com os critérios de indicação e recomendações no Brasil e cabe às instituições seguirem essas regras. A importância da avaliação de tratamentos envolve a garantia de qualidade dos seus usos nas instituições, tanto pela indução do método, quanto pela indicação correta.

A APA preconiza a utilização da ECT como tratamento de primeira escolha nos casos de necessidade de remissão dos sintomas agudos de forma rápida, em que os riscos de outros tratamentos são maiores do que os da ECT, de refratariedade ao tratamento medicamentoso ou boa resposta prévia à ECT ou quando é a terapêutica de escolha do paciente. Ademais, a utilização como tratamento de segunda escolha deve ocorrer, segundo a APA, quando há refratariedade da terapêutica adequada, aparecimento de efeitos colaterais graves ou mais exacerbados do que os provocados pela ECT e piora do quadro.
De acordo com a resolução 1640/2002 do Conselho Federal de Medicina (CFM) ${ }^{5}$, o uso da ECT deve ser feito, após a avaliação do estado clínico, exclusivamente pelo médico em ambiente hospitalar. É obrigatória a execução da anestesia (Resolução CFM 1363/1993). Conforme os preceitos bioéticos, o paciente ou a família, se necessário, deve preencher o consentimento informado, podendo o médico aplicar a terapia em casos de impossibilidade de obtenção de seu preenchimento. Nesse caso, o mesmo torna-se responsável pelo ato. Não há, no entanto, um protocolo padronizado pelo CFM para o número de aplicações da ECT, estando a manutenção do tratamento a critério da avaliação do paciente após cada sessão.

Por ser um método ainda experimental, baseado na observação clínica, a ECT precisa de parâmetros para se manter eficaz e segura ${ }^{6}$. Para isso, é relevante avaliar o tempo de convulsão, a quantidade de aplicações, o perfil dos pacientes (idade, sexo e diagnóstico) e dados como taxa de mortalidade e complicações.

O Instituto de Psiquiatria da Universidade Federal do Rio de Janeiro (IPUB-UFRJ) faz uso da ECT desde 1946. Todavia, apenas poucos trabalhos foram realizados com tal tema nessa instituição: Nardi \& Figueira $^{7}$ fizeram um levantamento epidemiológico dos períodos de 1961-1965 e de 1979-1983, Miranda et al. ${ }^{8}$ buscaram fazer uma avaliação crítica e qualitativa do seu uso e Alves et al. ${ }^{9}$ avaliaram o perfil demográfico e clínico de um grupo de pacientes do Instituto. Portanto, há quase 20 anos existe uma evidente necessidade de avaliação atual para a manutenção da instituição como um centro de referência brasileira na formação de profissionais, em equivalência aos padrões mundiais.

Avaliou-se o perfil do paciente que realiza ECT no IPUB-UFRJ e o critério de utilização da mesma nesse centro, atentando às indicações, à metodologia e aos resultados e comparando os pacientes antes e após o tratamento. Além disso, foram feitas comparações com dados obtidos em oito países (Estados Unidos, Austrália, Alemanha, Hungria, Japão, Índia, Rússia e Bélgica) e em outros artigos brasileiros.

\section{Método}

Foram colhidas informações de pacientes da enfermaria e do ambulatório do IPUB-UFRJ no período de junho de 2005 a junho de 2007, compreendendo um 
total de 69 pacientes. Foram consultados os bancos de dados da PubMed ${ }^{10}$ e da Capes $^{11}$ para a pesquisa dos artigos científicos disponíveis, envolvendo estudos epidemiológicos retrospectivos sobre o uso da ECT no Brasil, em outros países e no próprio IPUB-UFRJ em português, inglês, francês e alemão.

Foi feita uma pesquisa retrospectiva desse período, restrita a dados epidemiológicos referentes a sexo, idade, número de aplicações de ECT realizadas no paciente, diagnóstico de acordo com a décima revisão da Classificação Estatística Internacional de Doenças e Problemas Relacionados à Saúde (CID-10) e indicação à ECT. Foram selecionados todos os pacientes que realizaram ECT no período analisado.

\section{Resultados}

Todas as aplicações relativas aos 69 pacientes foram bilaterais e utilizaram propofol ou alfentanil como anestésicos e succinilcolina como relaxante muscular.
A clonidina foi utilizada nos pacientes hipertensos para serem evitados possíveis picos hipertensivos durante as sessões. O aparelho utilizado foi da marca comercial EMAI. Os efeitos colaterais foram brandos e transitórios, tais como amnésia anterógrada, desorientação e cefaléia. Mialgia, náuseas e fadiga foram raros. A taxa de morte foi nula.

O protocolo de sessões no IPUB-UFRJ é feito da seguinte forma: são realizadas três primeiras aplicações em dias consecutivos. Posteriormente, são feitas até oito aplicações em dias alternados. As aplicações seguintes são realizadas de duas a três vezes por semana. Obtémse uma média de oito aplicações por paciente. Interrompe-se a série quando o paciente se recupera dos sintomas agudos do transtorno.

Foi encontrada uma preponderância de pacientes do sexo feminino na realização da ECT, representando $71 \%$ do total (49 mulheres) frente a $29 \%$ do sexo masculino (20 homens). Foi verificado, ainda, um maior número de mulheres em todas as entidades nosológicas, isoladamente (Tabela 1).

Tabela 1 - Números absolutos de mulheres e homens em relação aos diagnósticos da CID-10

\begin{tabular}{lcc}
\hline Diagnósticos de acordo com a CID-10 & Sexo feminino & Sexo masculino \\
\hline F20 (esquizofrenia) & 22 & 12 \\
F31 (transtorno afetivo bipolar) & 16 & 3 \\
F33.3 (episódio atual grave com sintomas psicóticos) & 3 & 1 \\
F32.1 (episódio depressivo moderado) & 4 & 3 \\
F29 (psicose não-orgânica não especificada) & 3 & 1 \\
F30.2 (mania com sintomas psicóticos) & 1 & 0 \\
\hline
\end{tabular}

CID-10 = Classificação Estatística Internacional de Doenças e Problemas Relacionados à Saúde, $10^{\mathrm{a}}$ revisão.

A Tabela relaciona o diagnóstico do paciente segundo a CID-10 e seu sexo. Observa-se uma predominância do sexo feminino em todos os diagnósticos.

Em relação ao diagnóstico segundo a CID-10, pôdese observar uma prevalência da esquizofrenia (F20), que compreendeu 34 dos 69 pacientes (aproximadamente $49,3 \%$ ). Os outros diagnósticos encontrados foram o transtorno afetivo bipolar (F31) em 27,5\%; o episódio depressivo moderado (F32.1) em 10,1\%; o transtorno depressivo recorrente, episódio atual grave com sintomas psicóticos (F33.3) em 5,8\%; a psicose nãoorgânica não especificada (F29) em 5,8\% e a mania com sintomas psicóticos (F30.2) em 1,45\% do total dos pacientes, todos com valores aproximados (Figura 1).

Quanto às indicações para uso da ECT, 22,5\% foram por heteroagressividade, $15,7 \%$ por tentativa de suicídio, $13,5 \%$ por auto-agressividade, $13,5 \%$ por refratariedade aos psicofármacos, $9 \%$ por esquizofrenia grave, $9 \%$ por depressão grave, $6,7 \%$ por mania grave, $6,7 \%$ por catatonia e $3,4 \%$ por gravidez. Cabe observar que, em alguns casos, houve mais de uma indicação, tendo um total de indicações igual a 89 (Figura 2).

Em relação à idade dos pacientes, observou-se que $20,3 \%$ estavam na faixa etária de 18 a 29 anos, 31,9\% de 30 a 39 anos e $47,8 \%$ acima de 40 anos (valores aproximados). A média de idade dos pacientes foi de 41,3 anos $(\mathrm{p}=13,79)$.

Analisando o número de ECT realizadas por cada paciente, notou-se que em $34,8 \%$ dos casos foram aplicadas menos de cinco sessões; em $33,3 \%$, entre cinco a nove; em $15,9 \%$, entre 10 a 14; em 7,3\%, entre 15 a 19 ; e em $8,7 \%$ dos casos, mais de 20 sessões, aproximadamente (Figura 3). 


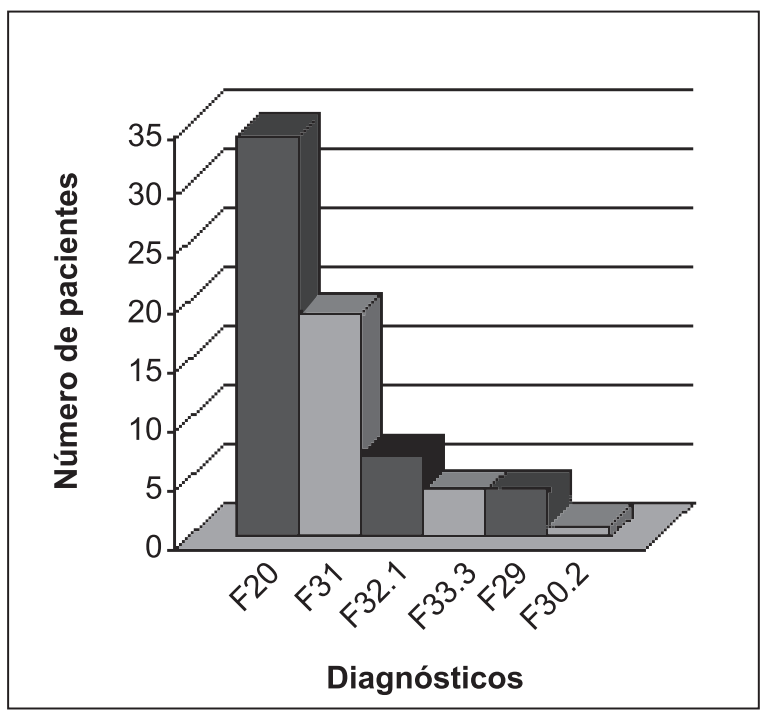

F20 = esquizofrenia; F29 = psicose não-orgânica não especificada; F30.2 = mania com sintomas psicóticos; F31 $=$ transtorno afetivo bipolar; F32.1 = episódio depressivo moderado; F33.3 = transtorno depressivo recorrente, episódio atual grave com sintomas psicóticos.

Figura 1 - Diagnósticos dos pacientes envolvidos na pesquisa, de acordo com a CID-10

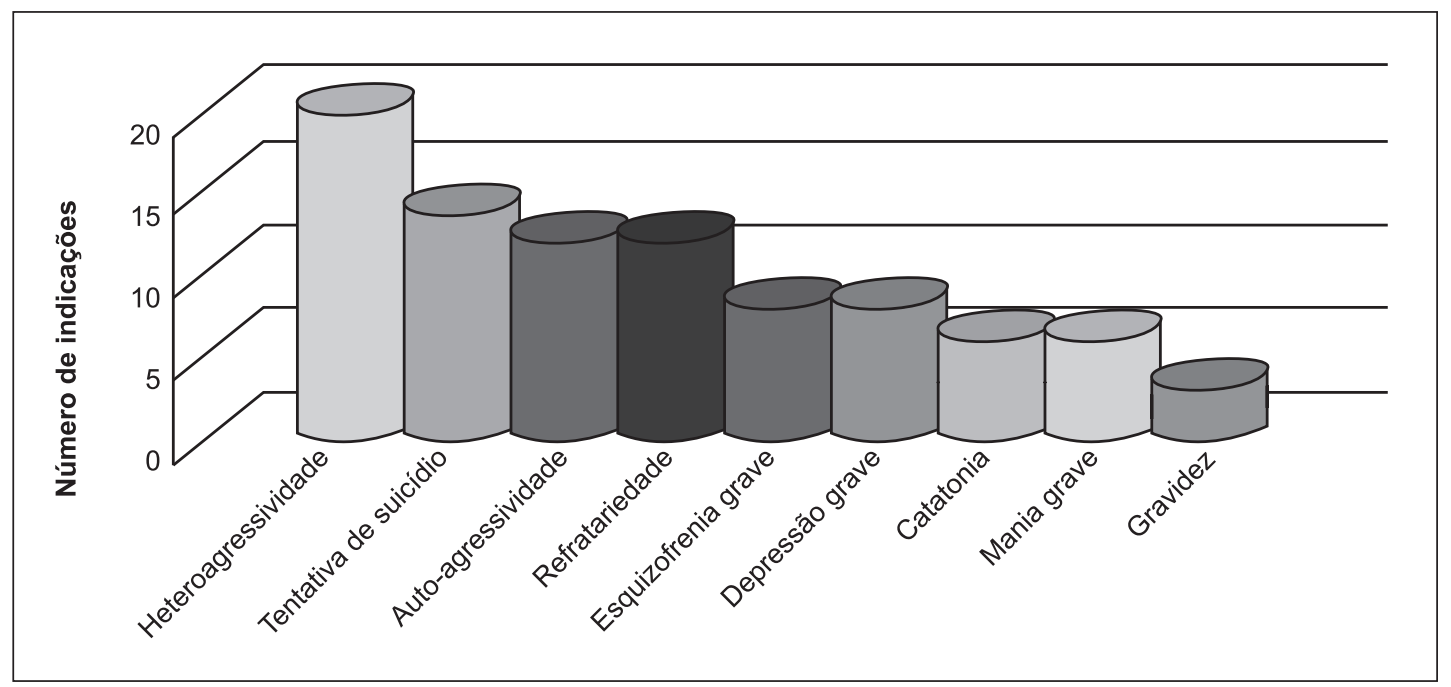

Figura 2 - Indicações clínicas para a realização de eletroconvulsoterapia

\section{Discussão}

Os artigos que fizeram uma análise epidemiológica semelhante a este no IPUB-UFRJ coletaram dados entre $1961-1965^{7}, 1979-1983^{7}, 1994-1995^{8}$ e $2003^{9}$. No primeiro período em questão, foi encontrada uma relação de 1:1,6 entre homens e mulheres submetidos a ECT, com um número de doses pouco homogêneo, que variou de uma a 20 aplicações. Também foi constatado que a esquizofrenia era o diagnóstico mais indicado para essa terapia. No período entre 1979-1983, a relação homem:mulher passou para 1:1,9, e as doses tornaramse mais homogêneas, tendo uma maioria de casos entre cinco e oito aplicações. O diagnóstico mais indicado para o tratamento continuou sendo a esquizofrenia. De abril de 1994 a agosto de 1995, foi encontrada uma 


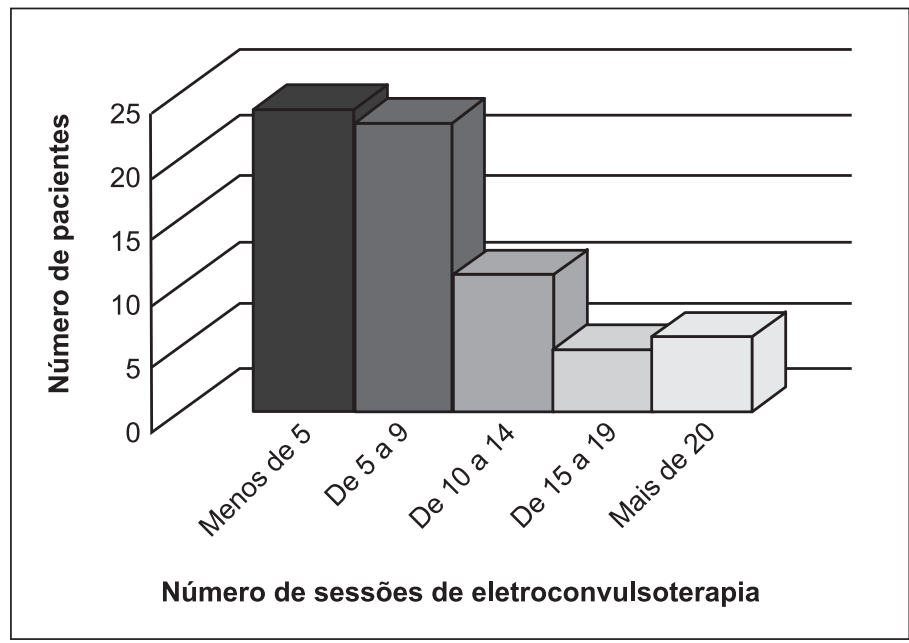

Figura 3 - Número de sessões de eletroconvulsoterapia realizadas por paciente (notar que a maioria dos pacientes realiza até 9 sessões)

relação homem:mulher mais alta que as anteriores: 1:2,7. A esquizofrenia foi, ainda, o diagnóstico de maior indicação no período; porém, dessa vez, houve um aumento nos diagnósticos de transtornos do humor. Obteve-se uma média de seis sessões por paciente e uma variação de idade com predomínio de 25 a 30 anos. No último período, foi feita uma análise de 32 pacientes, e se obteve uma relação homem:mulher de 1:1,9, e o diagnóstico de maior prevalência na indicação para a ECT foi o de transtorno bipolar do humor.

Um estudo conduzido por Volpe et al. no período de 1996 a 2000 em um hospital privado de Belo Horizonte, Minas Gerais ${ }^{12}$, continha uma amostra de 269 pacientes internados com episódio maníaco ou misto. Nesse estudo, observou-se uma freqüência de uso da ECT de 33,2\% em relação a outros tratamentos.

$\mathrm{Na}$ Europa, pesquisas encontraram como maior diagnóstico a depressão ${ }^{13-15}$ (Bélgica, 89,7 e $88 \%$; Alemanha, 44,3\%), com exceção da Hungria ${ }^{16}$, em que se encontrou a esquizofrenia (40,4\%). A média de ECTs realizadas nesse país foi de 9,3 sessões por paciente, tendo um valor próximo ao da Rússia ${ }^{17}$ ( 8,1 sessões) e ao da Alemanha ${ }^{15}$ (10,7 sessões).

$\mathrm{Na}$ Austrália, uma pesquisa envolveu 1.535 pacientes, e houve uma prevalência de $62,8 \%$ do sexo feminino em relação ao masculino. O diagnóstico de depressão foi de altíssima indicação para o tratamento $(75,2 \%)$. A média de aplicações foi de 12 para os transtornos afetivos e de 4,5 para a esquizofrenia. A faixa etária dos pacientes envolvidos é de $32,9 \%$ para maiores de 64 anos ${ }^{18}$.

Outro estudo ${ }^{19}$ realizado na Austrália avaliou 1.469 pacientes. Foi encontrado um predomínio de transtornos afetivos em relação aos demais transtornos. A maioria dos pacientes fez apenas uma sessão de ECT $(61,6 \%)$ e também era do sexo feminino $(65 \%)$. A faixa etária dominante entre os pacientes foi a de 19 a 64 anos (70,7\%), com uma idade média de 50 anos $(\mathrm{p}=18,85)$.

No Japão, 1.210 pacientes foram analisados, sendo observada uma maioria de mulheres $(53,7 \%)$, com idade entre 45 e 64 anos (40,4\%). A média de ECTs realizadas no período foi de 9,1 , e o diagnóstico mais comum foi o de esquizofrenia ${ }^{20}$.

$\mathrm{Na}$ Índia, obteve-se o maior número de pacientes analisados (19.632), e, diferente de todos os outros estudos, a média de homens foi maior que a de mulheres $(1,6: 1)$. Isso pode ser devido a uma maior prevalência de transtornos do humor em mulheres e uma maior internação com tratamento por ECT de pacientes com outros diagnósticos, como esquizofrenia. Assim como o Japão, a esquizofrenia foi o diagnóstico mais encontrado nesse país, e a faixa de idade está entre 45 e 64 anos. Foram feitas 5,4 sessões por paciente, em média $^{21}$.

Nos Estados Unidos, foi feita uma observação do mesmo tipo na Califórnia, entre 1977 e 1983, abrangendo um total de 18.627 pacientes. Dentre esses, 69\% são do sexo feminino, com idade superior a 25 anos e predomínio acima dos 45 anos $^{22}$. Em Nova Iorque, o estudo foi feito em três períodos: 1961-1965, 1966-1970 e 1971-1975. Em todos eles, encontrou-se a depressão como o diagnóstico mais prevalente. Em relação ao sexo predominante, foram encontradas variações nos resultados: nos primeiros anos de estudo, houve uma relação homem:mulher de 1:2,5; na segunda análise, essa relação caiu para 1:2 e, na última, para 1:1,723.

Um estudo realizado pela Mayo Clinic Rochester (Minnesota, USA) revelou que 87,3\% dos pacientes em 
uso da ECT responderam "Eu estou feliz por ter recebido ECT" em um questionário aplicado em 24 pacientes ${ }^{24}$. Isso é extremamente relevante para esta pesquisa, na medida em que mostra que o tratamento em questão é bem aceito pelo paciente e tem bons resultados para o mesmo.

O IPUB-UFRJ é a única instituição pública a realizar ECT no estado do Rio de Janeiro, assistindo pacientes provindos das mais variadas localidades. Representa, portanto, um bom modelo de estudo do perfil fluminense.

A maior prevalência de diagnósticos de internação nesse centro psiquiátrico é de esquizofrenia, seguida de transtorno afetivo bipolar. Por ser um hospital terciário, o IPUB-UFRJ interna os casos mais graves, como esquizofrenia em fase aguda e refratária aos medicamentos. Isso se reflete no padrão diagnóstico dos pacientes encaminhados para a realização da ECT, como observado nos resultados desta pesquisa.

Na revisão de Salleh et al. ${ }^{6}$, concluiu-se, a partir de estudos e relatos da literatura, que a manutenção das medicações durante as sessões de ECT apresenta resultados mais rápidos do que seu uso isolado. Isso acarreta uma vantagem terapêutica, garante a segurança dos pacientes e dos profissionais de saúde, assim como facilita a conduta na enfermaria. É relevante fazer uma ressalva aos medicamentos que não são prescritos em concomitância à ECT, como os antidepressivos inibidores da monoaminoxidase, o carbonato de lítio e os anticonvulsivantes. Ademais, o IPUB-UFRJ, sendo um órgão gratuito, recebe um número considerável de pacientes trazidos por terceiros, após causarem prejuízo à ordem pública ou a si próprios. Acreditamos que tais comportamentos ofensivos estejam relacionados ao perfil do paciente internado nesse tipo de entidade. Os achados desta amostra corroboram esse raciocínio, já que a heteroagressividade, a tentativa de suicídio e a auto-agressividade foram encontradas como indicações mais freqüentes para a realização da ECT.

Verificou-se um predomínio do sexo feminino tanto de forma absoluta quanto relativa aos diagnósticos separadamente. Constatou-se, ainda, que os pacientes diagnosticados, de acordo com a CID-10, como episódio atual grave com sintomas psicóticos e episódio depressivo moderado apresentam uma relação homem:mulher de $1: 3$ e $1: 1,3$. Isso pode ser compreendido na medida em que os transtornos citados são mais freqüentes em pacientes do sexo feminino ${ }^{25}$.

Ainda que a esquizofrenia ${ }^{26} \mathrm{e} o$ transtorno bipolar do humor ${ }^{27}$ não apresentem uma menor relação homem:mulher estatisticamente, encontramos uma elevada preponderância do sexo feminino para esses diagnósticos.

O presente estudo revelou uma relação total homem:mulher de 1:2,5. Tais resultados são compatíveis com os encontrados em algumas partes do mundo, como em Nova Iorque $(1: 2,5)^{23}$, Califórnia $(1: 2,2)^{22}$, Austrália $(1: 1,7 \text { e } 1: 1,8)^{18,19}$ e Brasil $(1: 1,9 \text { e } 1: 2,7)^{7,8}$. A Índia foi o único país a apresentar uma relação inversa a esta pesquisa $(1,6: 1)^{21}$. As pesquisas da Alemanha e da Rússia não apresentam dados sobre tal relação.

$\mathrm{Na}$ maioria dos casos $(49,3 \%)$, o diagnóstico foi esquizofrenia, com uma relação homem:mulher de 1:1,8. A provável razão para esse dado seria a maior prevalência de mulheres em relação a homens no Instituto.

Quanto aos achados relacionados ao diagnóstico, notou-se uma manutenção da superioridade percentual de esquizofrênicos no Instituto, como apresentado anteriormente por Nardi \& Figueira ${ }^{7}$ e Miranda et al. ${ }^{8}$. O mesmo padrão é observado em países como Japão ${ }^{20}$, Hungria $^{16}$ e Índia ${ }^{21}$. No entanto, houve uma discrepância nos resultados obtidos na Austrália ${ }^{18,19}$, Alemanha ${ }^{15}$, Bélgica $^{14,15}$ e nos EUA ${ }^{22,23}$, que demonstraram valores mais elevados para diagnósticos de depressão.

Pesquisando as indicações para o uso da ECT em diversos países, observou-se uma escassez de dados nesse âmbito, posto que muitos artigos consideram os diagnósticos as próprias indicações. Assim, a refratariedade foi a justificativa mais comum encontrada para a ECT em tais locais, divergindo da heteroagressividade, encontrada na presente amostra. Neste trabalho, adotou-se o critério "indicação" como fundamentação médica para a realização de tal terapia. Isso foi estabelecido por acreditarmos que o diagnóstico por si só é insuficiente como motivo de encaminhamento do paciente a esse tipo de tratamento.

Salleh et al. ${ }^{6}$ relataram que o número de sessões necessárias para o tratamento da depressão varia de seis a oito aplicações, podendo chegar a 12 nos casos mais graves. Tal dado confere com as médias encontradas na Rússia $^{10}$, Alemanha ${ }^{15}$, Austrália ${ }^{18,19}$ e EUA ${ }^{22,23}$, onde os diagnósticos mais comuns são os transtornos relativos à depressão.

Em relação à faixa etária dos pacientes submetidos a ECT, houve uma concordância entre o perfil mundial e o de nossa entidade: um maior contingente de pacientes com idade superior a 40 anos. Na amostra, essa categoria corresponde a $47,8 \%$, característica essa semelhante no Japão ${ }^{20}(40,4 \%)$ e na Índia ${ }^{21}(43,9 \%)$.

Nos países em que há preponderância de depressão ${ }^{18,19,23,24}$, a faixa etária dos pacientes que realizam ECT é razoavelmente mais alta que a do Brasil $^{7,8,12}$, do Japão ${ }^{20}$ e da Índia ${ }^{21}$, onde foi encontrada maior prevalência diagnóstica de esquizofrenia. Isso pode ser entendido pelo acometimento mais precoce da esquizofrenia em relação à depressão.

As limitações do nosso estudo envolvem o fato de contarmos com uma amostra pequena de pacientes e com um curto período de análise. Além disso, cabe 
observar que a avaliação da evolução dos pacientes em relação ao tratamento é subjetiva, já que depende da observação clínica do médico, o que traz mais uma restrição ao trabalho, em termos de objetividade de avaliação da ECT como método.

\section{Conclusões}

Este estudo avalia o uso da ECT como tratamento no IPUB-UFRJ. Todas as aplicações estão de acordo com os critérios e recomendações da Associação Mundial de Psiquiatria ${ }^{6}$ e com o modelo utilizado nos países comparados.

Embora seja um tema ainda polêmico, o uso da ECT persiste como um bom método para remissão de sintomas graves, como os dos pacientes indicados em nosso artigo.

O presente trabalho revela uma manutenção geral dos padrões da utilização dessa terapêutica no IPUBUFRJ para as categorias avaliadas, qualitativamente. $\mathrm{Na}$ medida em que há resultados consideravelmente destoantes entre o número de sessões aplicadas a cada paciente nos países analisados, fica a impressão de que a manutenção das sessões de ECT para o paciente é feita por avaliações subjetivas do médico. Dessa maneira, evidencia-se uma ausência de modelo comum de utilização do método. Isso reforça a necessidade de uma gama de estudos direcionados ao estabelecimento do padrão de aplicações necessárias à boa resposta dos pacientes submetidos ao tratamento.

\section{Agradecimentos}

À enfermeira Rita de Simões; ao secretário do CIPE, Sr. Dario; à secretária do faturamento, Cíntia; ao secretário do ambulatório, Sr. Álvaro e a todos os pacientes que contribuíram para esta pesquisa direta ou indiretamente.

\section{Referências}

1. Stone MH. Healing the mind, a history of psychiatry from antiquity to the present. New York: WW Norton; 1997. p. 139-56.

2. Lisanby SH. Electroconvulsive therapy for depression. N Eng J Med. 2007;357(19):1939-45.

3. American Psychiatric Association. The practice of electroconvulsive therapy: recommendations for treatment, training, and privileging. A task force report of the American Psychiatric Association. 2nd ed. Washington: American Psychiatric Association; 2001.
4. Volpe FM. Eletroconvulsoterapia para episódios maníacos. Associação Brasileira de Psiquiatria. Boletim Científico, edição 12 [cited 08-09-2008]. http://www.abpbrasil.org.br/medicos/boletim/ exibBoletim/?bol_id=12\&boltex_id=46

5. Brasília, Conselho Federal de Medicina. Resolução CFM No $1.640 /$ 2002, jul 2002. [cited 08-09-2008]. http:// www.portalmedico.org.br/resolucoes $/ \mathrm{cfm} / 2002 / 1640$ 2002.htm

6. Salleh MA, Papakostas I, Zervas I, Christodoulou G. Electroconvulsoterapia: critérios e recomendações da Associação Mundial de Psiquiatria. Rev Psiq Clin. 2006;33(5):262-7.

7. Nardi AE, Figueira ILV. A eletroconvulsoterapia no Instituto de psiquiatria - UFRJ: períodos - 1961-1965 e 1979-1983. J Bras Psiquiatr. 1988;37(5):241-6.

8. Miranda CML, Costa FA, Santos LA, Menezes SJ. Uma análise crítica quanto às condições que envolvem a indicação da terapia por eletrochoque em pacientes psiquiátricos internados. J Bras Psiquiatr. 1998;47(2):67-72.

9. Alves CE, Fontenelle LF, Cruz B, Brasil MA. Características clínicas e demográficas de um grupo de pacientes em uso de eletroconvulsoterapia. J Bras Psiquiatr. 2005;54(2):90-3.

10. National Center for Biotechnology Information. [cited 08-09-2008]. Available from: http://www.ncbi.nlm.nih.gov/pubmed/

11. Coordenação de Aperfeiçoamento de Pessoal de Nível Superior (CAPES). [cited 08-09-2008]. Available from: http:// www.capes.gov.br/

12. Volpe FM, Tavares A, Correa H. Naturalistic evaluation of inpatient treatment of mania in a private Brazilian psychiatric hospital. Rev Bras Psiquiatr. 2003;25(2):72-7.

13. Sienaert P, Filip B, Willy M, Joseph P. Electroconvulsive therapy in Belgium: a questionnaire study on the practice of electroconvulsive therapy in Flanders and the Brussels Capital region. J ECT. 2005;21(1):2-6.

14. Sienaert P, Dierick M, Degraeve G, Peuskens J. Electroconvulsive therapy in Belgium: a nationwide survey on the practice of electroconvulsive therapy. J Affect Disord. 2006;90(1):67-71.

15. Baghai TC, Marcuse A, Möller HJ, Rupprecht R. [Electroconvulsive therapy at the Department of Psychiatry and Psychotherapy, University of Munich. Development during the years 1995-2002]. Nervenarzt. 2005;76(5):597-612.

16. Gazdag G, Kocsis N, Lipcsey A. Rates of electroconvulsive therapy use in Hungary in 2002. J ECT. 2004;20(1):42-4.

17. Nelson AI. A national survey of electroconvulsive therapy use in the Russian Federation. J ECT. 2005;21(3):151-7.

18. Wood DA, Burgess PM. Epidemiological analysis of electroconvulsive therapy in Victoria, Australia. Aust N Z J Psychiatry. 2003;37(3):307-11.

19. Teh SP, Xiao AJ, Helmes E, Drake DG. Electroconvulsive therapy practice in Western Australia. J ECT. 2005;21(3):145-50.

20. Chanpattana W, Kojima K, Kramer BA, Intakorn A, Sasaki S, Kitphati R. ECT Practice in Japan. J ECT. 2005;21(3):139-44.

21. Chanpattana W, Kunigiri G, Kramer BA, Gangadhar BN. Survey of the practice of electroconvulsive therapy in teaching hospitals in India. J ECT. 2005;21(2):100-4.

22. Kramer BA. Use of ECT in California, 1977-1983. Am J Psychiatry. 1985;142(10):1190-2.

23. Babigian HM, Guttmacher LB. Epidemiologic considerations in electroconvulsive therapy. Arch Gen Psychiatry. 1984;41(3):24653.

24. Goodman JA, Krahn LE, Smith GE, Rummans TA, Pileggi TS. Patient satisfaction with electroconvulsive therapy. Mayo Clin Proc. 1999;74(10):967-71.

25. Grigoriadis S, Robinson GE. Gender issues in depression. Ann Clin Psychiatry. 2007;19(4):247-55.

26. Keller MB. Prevalence and impact of comorbid anxiety and bipolar disorder. J Clin Psychiatry. 2006;67 Suppl 1:5-7.

27. Maier W. Common risk genes for affective and schizophrenic psychoses. Eur Arch Psychiatry Clin Neurosci. 2008;258 Suppl $2: 37-40$ 